\title{
Research on Functions and Techniques of Special Sound in Guzheng Performance
}

\author{
Peng Shanxia \\ Linyi University, Linyi, Shandong, China \\ Pengshanxia001@163.com
}

Keywords: Special sound; Functions and techniques; Guzheng performance

\begin{abstract}
Guzheng is a treasure of Chinese national musical instruments. Special sound in Guzheng performance is the essence of the Guzheng art. This paper firstly gives the basic concepts of Guzheng performance and special sound, and then points out the important role of special sound effects and it's techniques in Guzheng playing, finally puts forward the development trend of special sound effects in order to provide references for relevant researchers.
\end{abstract}

\section{Introduction}

As a treasure of Chinese national musical instruments, Guzheng has a long history. It has been passed down in the early spring and autumn period, dating back nearly 3000 years. The cultural vein of Guzheng art and ancient Chinese to traditional culture and arts is the come down in one continuous line. When the sound block western orchestras thick in the ears, people feel the shock, are listening to music, but make people feel a kind of quiet filling. Due to its fine and deep, we get a very different aesthetic feeling from western music.

The sound effects mean the results or effects made by the sound. It can enhance the sense of reality, atmosphere or play information, voice into the sound of the sounding body items. Zither sound performer refers to the use of various techniques, tools anywhere in the body of the Guzheng, manufacturing is different from the traditional playing technique produced sound effect. Such as: take the city board of the piano with the palm of your hand or nail slapping, strings in the long sound, sound in a disordered high code and sweeping strings, playing the left button shake. We will make a general summary of the special sound effect in recent years Guzheng playing and analyze the techniques of it to provide some references to the Guzheng performer.

\section{Functions of Special Sound in Guzheng Performance}

Functions in Different Periods. From 1950s to 1970s, Chinese Guzheng music creators or performer is the main body of the playing. The special sound during this period mainly contained simulation of nature of the sound and sound of artificial simulation has become popular elements of Guzheng creation during this period. A simulation of the sound of gongs and drums and other artificial sound, special effects such as point column playing, playing techniques. At the time, and for playing multi folk gatherings into celebration, so the sound of gongs and drums to simulate special sound effects to create a festive atmosphere, foil the cheerful rhythm role in Guzheng playing. There are simulation of the wind, the sound or the sound of wind and rain and other natural sounds, such as the special sound playing buckle shake, the technique of blowing disorderly pitch. Because of these skills in playing a more intensive plucked frequency, in the emotional expression is more intense, so in music often show a dangerous situation or swift. From 1970s to 1990s, especially in the 1980s, professional composers joined the Guzheng music creation team. They use two party composition techniques combined with traditional Guzheng Chinese tone to create a large number of novel Guzheng works. These outstanding works based on Inheritance and development of traditional, adding new ideas and boldly innovate, to rewrite the thousands of years of inheritance is not deviant, but definitely not slavishly follow. In this process, a special sound Guzheng playing the role cannot be ignored. The smooth progress of Guzheng art is no longer just the pursuit of melodious rhythm and technical difficulty, but development began to focus on the expression of 
ideas and new timbre acoustics. The special sound of this period began to break through the simulation of artificial sound or nature sound simple, and musical fusion is more natural, sometimes it's just to use rendering mood, rhythm and harmony of the prominent or traditional stereo contrast. The use of playing techniques such as pitch rattling, disorder, no longer single, using a combination of various techniques on pitch tone according to accurate performance, disorderly strings sequence has greatly increased the creation of music fun. The generous use of special sound in Guzheng performance provides more space for the creation of performer, making each performer different.

Functions in Music Creation. The use of special sound effect has been greatly developed, but until now, people still continue to explore the special sound effects. When it just appeared, any new things are very vulnerable to opposition from the people and do not understand, including the use of a special sound of playing the zither. When a special sound has just started, because the performance effect with the traditional Guzheng, special sound with sound playing effects therefore be quite different, by many people criticized the Guzheng and criticism. With the continuous development of special sound, creative zither music art has been greatly expanded. The final development of the Guzheng music plays an effective role in promoting. Now a lot of Guzheng performers and composers in Guzheng music creation are on the special sound playing effect affirmed and supported, and has carried on the positive use, which fully shows the positive role in the development of special sound effects to the Guzheng. From the beginning to continued growth in the process, using the special sound effect has been in innovation and development, effectively promoting the progress of Guzheng music art. Therefore, in the future development of the road zither music, zither playing the sound will continue to develop continuously, but also in front of an audience showing a more colorful attitude, eventually to enrich the great Guzheng music sound, and effectively promote the development of Guzheng art.Compared with the traditional Guzheng sound, using special sound effects not only depict the image of music plays an important role, but also helps the musician to create good compositions.

\section{Techniques of Special Sound in Guzheng Performance}

We can divide the special sound in Guzheng performance into three categories according to the methods of performance. They are slapping, strumming and friction. Almost all of the special effects can be summed up into the three categories.

Slapping.The beat and rush in instrument performance is called slapping. It is the simulation of percussion instruments in the use of techniques in the Guzheng playing techniques. There are so many kinds of percussion music culture has a history of thousands of years in China, whether it is in the form of performance, or playing techniques, even in their own music creation are very precious cultural wealth. When playing in the process not only to the percussion sound effects to be effectively simulated on Rattling skills, but also need to pay attention to their own body language. Body language in the process of playing percussion is very important, but the routine is also very complete. But when the Guzheng is still not perfect in the specific application, so in the slap technique from the time must take its essence, and innovation, the maximum will eventually play a good physical performance. In the process of playing Guzheng music can be divided into two types on techniques, namely combination type and single type. The main types of slap strings, the strings are rattling slap for part of the string, so that the sound block effect can be produced. Often need to combine the body to be able to slap the strings on the effect of play techniques. But in some music often will slap strings for atmosphere or techniques will lead. At the same time also has a slap and slap the body somewhere code techniques. There are good connections of several slapping in a lot of Guzheng music creation.

Strumming. Strumming means the strings with the fingers or a plectrum. There are ancient Guzheng instruments whichplay a decisive role in national musical instruments. In modern Guzheng music, traditional stringed tone has been not able to meet the composer's intention, is not enough to express the contemporary more complex behind the creation of thought, the new application of plucking techniques will emerge as the times require. According to the fingers or plectrum plucked strings is called plucked, Guzheng in the national instrument in China for 
thousands of years of history which always occupies a very important position. The traditional plucked in the modern Guzheng music timbre is often unable to make the composer's creation will be fully satisfied, but also with distinctive characteristics of the times combined, so in the art performance has limitations, and it is the use of new techniques ". Now many composers in the creation of music when the love will be left as a regional code of "clearing", the use of special sound code left area playing out the random and messy characteristics, but also has the characteristics of grotesque, clever and gruesome, composers in the new period of code left the use of the most incisive area. As the common Guzheng performance techniques, blowing techniques are widely recognized by composers, the technique of using very fast scale playing way can make people feel very curious features. With the continuous development of Chinese art of Guzheng, Guzheng artist improved traditional strumming techniques, thus playing special sound blowing techniques can produce. Special sound strumming in Guzheng performance can be divided into several types string strumming blowing and blowing left code. At the same time, the common Guzheng strumming techniques include bartok, sweeping strings, plucked sound, overtone.

Friction.There is the move back and forth in the object is called friction. Can be divided in special friction sound Guzheng in four, that is by friction and the emergence of nail strings, strings and voice by voice, palm friction appears by friction and bow strings and strings of voice by other items up to now the sound of friction. Compared with plucking techniques and friction techniques, in which the use of zither playing techniques of friction is relatively small, but it has a very important role. Bow skills has more application in Guzheng strings, which is mainly on the string bow skills for reference, and combining with the resonance box zither bigger and longer strings of advantage, and bow caused some unique sound effects in Guzheng strings, compared with the traditional Guzheng "point" like sound, the sound is completely different "line" sound like. To make full use of the bow technique, will only "connect the dots"Guzheng long play state has completely changed, mutual friction between the bows. In music playing on linear music make full use, not only as a plucked instrument Guzheng performance function is greatly expanded, but also the zither sound effects have been effectively enriched. The "Harvest Festival" performed by Guan Naizhong in 1983 applied national orchestral Techniques to bow to friction among Guzheng strings on the use for the first time. The friction in the composition can make full use of linear lines, bringing about unique listening experience to the audience.

\section{Tendency of Special Sound in Guzheng Performance}

It's not a reactionary of Guzheng music to appear the special sound in the Guzheng performance. Special effects in Guzheng playing techniques constantly enrich the process. The use of more and more widely is an inevitable trend of Guzheng music technology development trend of the times. As a kind of music language is very different with the traditional sound, playing music in the modern role of special sound effects in natural law under the new push Chen has been used effectively, it will not only more profound thought of modern performance, but also will be more diverse music image display. The end of China's traditional music performance practices expanding played an effective role in promoting. At the same time, the use of special effects in Guzheng playing should also experience the long-term test, which is also the most realistic portrayal for the continuous development of Guzheng music art. Guzheng playing special sound effect used so far is only the beginning, the first of its aims from emergence to the development of several decades, the use of Guzheng performer and composer who always takes a positive attitude in the development, improve the special sound of Guzheng music in. More composers and performers will be applied to the Guzheng music creation, which indicates that this group of special sound recognition. As the most professional them in the use of special sound effect, is not only to create a gimmick to drift, special sound effects as an innovative sound, compared with the traditional Guzheng sound, whether it is in the description of music image or in the expression of the creative ideas, are quite different functions, and the effect of different styles. In view of this, the writing group is undoubtedly the most favorable development of special sound impetus. As long as there are more 
excellent works with special sound effects are emerging, special sound will have unlimited space for Guzheng art development.

\section{References}

[1] X. Sheng. Research on the Creation Characteristics and the Performance Hints of Guzheng Solo Fragrant Jasmine, J.Journal of Huangshan University. 2(2010) 16 - 17.

[2] M. Xue. A Discussion on the Problems in Guzheng Performance Practice, J.Sichuan University of Arts and Science Journal. 5(2013) 35 - 37

[3] Y. Wang. The Development Trend of Guzheng in Contemporary Era, J.Journal of Yangtze University (Social Sciences). 6(2006) 106 - 108 